$07.1 ; 07.2 ; 07.3$

\title{
Формирование гетероструктур GaP/Si-фотопреобразователей с помощью комбинации методов МОС-гидридной эпитаксии и атомно-слоевого плазмохимического осаждения
}

\author{
(C) А.В. Уваров ${ }^{1,2}$, А.И. Баранов ${ }^{1,2}$, Е.А. Вячеславова ${ }^{1,2}$, Н.А. Калюжный ${ }^{3}$, Д.А. Кудряшов ${ }^{1,2}$, \\ А.А. Максимова ${ }^{1,2}$, И.А. Морозов ${ }^{1,2}$, С.А. Минтаиров ${ }^{3}$, Р.А. Салий ${ }^{3}$, А.С. Гудовских ${ }^{1,2}$ \\ ${ }^{1}$ Санкт-Петербургский национальный исследовательский Академический университет им. Ж.И. Алфёрова РАН, \\ Санкт-Петербург, Россия \\ ${ }^{2}$ Санкт-Петербургский государственный электротехнический университет „ЛЭТИ“, Санкт-Петербург, Россия \\ ${ }^{3}$ Физико-технический институт им. А.Ф. Иофрфе РАН, Санкт-Петербург, Россия \\ E-mail: lumenlight@mail.ru
}

Поступило в Редакцию 24 марта 2021 г.

В окончательной редакции 26 апреля 2021 г.

Принято к публикации 27 апреля 2021 г.

\begin{abstract}
Впервые показана возможность создания нижнего перехода многопереходных $\mathrm{A}_{3} \mathrm{~B}_{5} / \mathrm{Si}$ солнечных элементов на основе гетероструктуры $n-\mathrm{GaP} / p-\mathrm{Si}$, выращенной с помощью комбинации технологий атомнослоевого плазмохимического осаждения и металлоорганической газофазной эпитаксии при температуре $T_{s}$, не превышающей $650^{\circ} \mathrm{C}$. Фотоэлектрические свойства структур, выращенных при $T_{s} \leq 650^{\circ} \mathrm{C}$, зависят от условий процесса, в частности от использования дополнительной обработки в плазме $\mathrm{Ar}$.
\end{abstract}

Ключевые слова: фосфид галлия, кремний, солнечный элемент.

DOI: 10.21883/PJTF.2021.14.51189.18781

На данный момент одним из наиболее перспективных направлений дальнейшего развития солнечной энергетики является создание монолитных многопереходных фотоэлектрических преобразователей на основе интеграции соединений $\mathrm{A}_{3} \mathrm{~B}_{5} \mid$ и кремния. Кремний исходя из совокупности факторов является одним из наиболее подходящих материалов нижнего перехода для создания двухпереходного солнечного элемента (СЭ) [1]. Теоретическое значение КПД для структуры $\mathrm{GaAs}_{0.78} \mathrm{P}_{0.22} / \mathrm{Si}$ может превышать $32 \%$ [2]. Однако для создания монолитных СЭ на подложке $\mathrm{Si}$ необходимо вырастить буферный слой, согласованный по параметру кристаллической решетки с $\mathrm{Si}$. Самым подходящим материалом для формирования буферного слоя является $\mathrm{GaP}$, имеющий рассогласование параметров кристаллической решетки на уровне $0.36 \%$ при комнатной температуpe [3]. Помимо этого GaP может играть роль как широкозонного окна, так и эмиттера для нижнего $p-n$-перехода на основе $\mathrm{Si}$. Немаловажным является то, что для $\mathrm{GaP}$ разрыв зон проводимости с $\mathrm{Si}$ coставляет всего $0.25 \mathrm{eV}$, что исключает возникновение нежелательных барьеров на интерфейсе гетероперехода $n-\mathrm{GaP} / p-\mathrm{Si}$ [4]. Возможность использования гетероперехода $n-\mathrm{GaP} / p$-Si в качестве СЭ подтверждается как результатами компьютерного моделирования [5], так и экспериментально [6]. Ранее структуры GaP/Si были сформированы с использованием металлоорганической газофазной эпитаксии (МОС-гидридной эпитаксии, MOVPE) [7] и молекулярно-пучковой эпитаксии (MBE) [8]. Однако методы получения слоев GaP требуют относительно высоких температур роста, по- скольку необходим предварительный отжиг подложки в ростовой камере для реконструкции поверхности и снятия оксида. Типичная температура снятия оксида с кремниевой подложки при росте методом MOVPE составляет $850-1000^{\circ} \mathrm{C}$ [9]. Требование отжига при высоких температурах усложняет создание интерфейса высокого качества в гетероструктурах $\mathrm{GaP} / \mathrm{Si}$. Было показано, что при осаждении GaP методом MBE в $\mathrm{Si}$ формируется дефектный слой, а при отжиге в камере значительно падает время жизни в кремниевой подложке из-за быстродиффундирующих примесей $[10,11]$. Таким образом, для формирования гетероперехода $\mathrm{GaP} / \mathrm{Si}$ необходимо использовать низкотемпературные методы, которые не приводят к образованию дефектов на интерфейсе.

В предыдущей работе была показана возможность формирования тонкого $(3-5 \mathrm{~nm})$ нуклеационного слоя $\mathrm{GaP}$ на поверхности $\mathrm{Si}$ при помощи низкотемпературного метода плазмохимического атомно-слоевого осаждения (PEALD) для последующего эпитаксиального роста с помощью MOVPE [12]. В настоящей работе представлены результаты исследований по созданию солнечных элементов на основе гетероперехода $n-\mathrm{GaP} / p$-Si, сформированного с помощью метода MOVPE на подложках Si c нанесенным методом PEALD нуклеационным слоем GaP.

В качестве подложки использовался кремний $p$-типа легирования $\left(10^{16} \mathrm{~cm}^{-3}\right)$ ориентации $(100)$ с разориентацией в $4^{\circ}$ в направлении плоскости (111). Непосредственно перед процессом осаждения с поверхности $\mathrm{Si}$ был удален слой естественного оксида с помощью 
Описание исследуемых образцов

\begin{tabular}{|c|c|c|c|c|c|c|}
\hline \multirow{2}{*}{$\begin{array}{l}\text { Номер } \\
\text { образца }\end{array}$} & \multicolumn{2}{|c|}{ PEALD } & \multirow{2}{*}{$\frac{\text { MOVPE }}{\text { Температура, }{ }^{\circ} \mathrm{C}}$} & \multicolumn{3}{|c|}{ Параметры } \\
\hline & Режим & $\begin{array}{c}\text { Количество } \\
\text { циклов }\end{array}$ & & $V_{o c}, \mathrm{~V}$ & $I_{s c}, \mathrm{~mA} / \mathrm{cm}^{2}$ & $f f, \%$ \\
\hline 1 & $\begin{array}{c}\text { in situ отжиг } \\
\text { в плазме Ar }\end{array}$ & 20 & 600 & 0.395 & 23.10 & 53.2 \\
\hline 2 & то же & 20 & 650 & 0.396 & 23.41 & 53.5 \\
\hline 3 & без Ar-плазмы & 40 & 600 & 0.415 & 23.00 & 56.0 \\
\hline 4 & то же & 40 & 650 & 0.447 & 25.33 & 50.2 \\
\hline
\end{tabular}

обработки в растворе HF. Нелегированные нуклеационные слои $\mathrm{GaP}$ толщиной $3-5 \mathrm{~nm}$ были осаждены при температуре $390^{\circ} \mathrm{C}$ методом PEALD в двух режимах: c in situ отжигом в плазме $\mathrm{Ar}$ и без использования плазмы Ar. При этом различная скорость осаждения в этих режимах была компенсирована количеством циклов для получения одинаковой результирующей толщины. Использование in situ отжига в плазме $\mathrm{Ar}$, с одной стороны, позволяет достичь лучших структурных свойств слоев $\mathrm{GaP}$, но, с другой стороны, может привести к формированию радиационных дефектов в приповерхностной области $\mathrm{Si}$ [13]. Эпитаксиальные слои $\mathrm{GaP} n$-типа проводимости $\left(\sim 10^{17} \mathrm{~cm}^{-3}\right)$ толщиной $300 \pm 30 \mathrm{~nm}$ были выращены методом MOVPE на исследовательской установке Aixtron AIX200/4 с реактором горизонтального типа при пониженном давлении $\left(100\right.$ mbar) при двух температурах: 600 и $650^{\circ} \mathrm{C}$. В обоих методах триметилгаллий $\left(\mathrm{Ga}\left(\mathrm{CH}_{3}\right)_{3}\right)$ использовался в качестве источников элементов третьей группы, а фосфин $\left(\mathrm{PH}_{3}\right)-$ в качестве источника элемента пятой группы (фосфора). В качестве источника легирующей примеси $n$-типа (кремния) использовался силан $\left(\mathrm{SiH}_{4}\right)$. Сверху с помощью фотолитографии формировалась контактная сетка на основе $\mathrm{Au} / \mathrm{Ge}$. Нижний контакт был сформирован за счет нанесения In. Для обеспечения минимального контактного сопротивления контакты отжигались при температуре $450^{\circ} \mathrm{C}$. Просветляющие покрытия на фоточувствительную область СЭ не наносились. В таблице представлены основные различия в условиях формирования исследуемых образцов, а также значения напряжения холостого хода $\left(V_{o c}\right)$, тока короткого замыкания $\left(I_{s c}\right)$ и фактора заполнения $(f f)$ для полученных структур.

Вольт-амперные характеристики изготовленных СЭ измерялись в стандартных условиях освещения $\left(\mathrm{AM} 1.5 \mathrm{G}, 100 \mathrm{~mW} / \mathrm{cm}^{2}, 25^{\circ} \mathrm{C}\right)$ с использованием имитатора солнечного излучения Abet Technologies SunLite и источника-измерителя Keithley 2400. Полученные вольт-амперные характеристики представлены на рис. 1.

Спектральные характеристики регистрировались при помощи измерительного стенда на основе монохроматора Solar Laser Systems M266. На спектрах внешней квантовой эффективности наблюдалась интерференционная картина, обусловленная разницей показателя

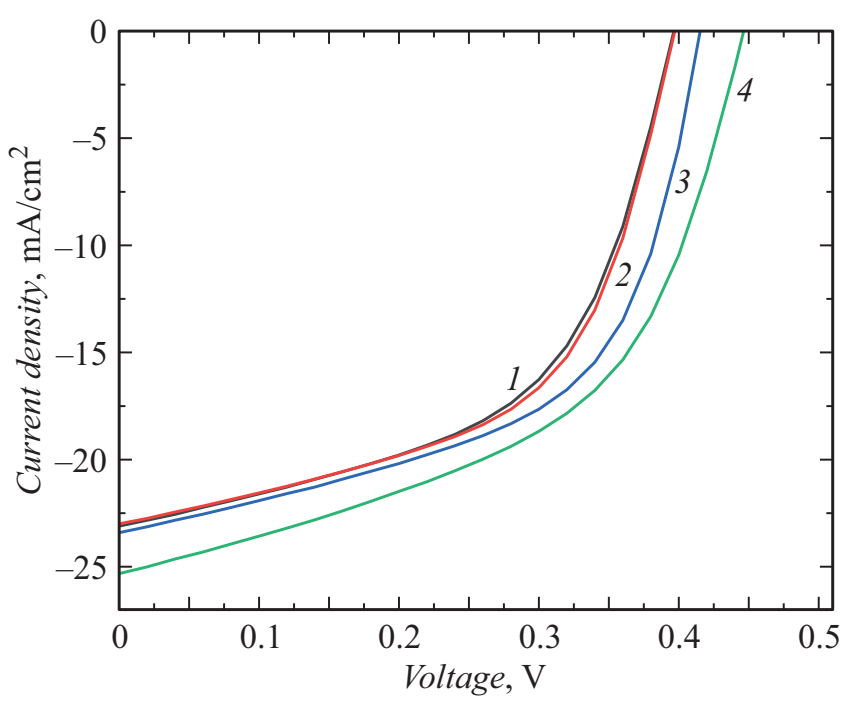

Рис. 1. Вольт-амперные характеристики СЭ в стандартных условиях освещения (АМ1.5G, $100 \mathrm{~mW} / \mathrm{cm}^{2}, 25^{\circ} \mathrm{C}$ ). Номера кривых соответствуют номерам образцов в таблице.

преломления $\mathrm{Si}$ и слоя $\mathrm{GaP}$. Чтобы исключить влияние интерференции на форму спектров квантовой эффективности, были измерены спектральные зависимости коэффициента отражения. Приведенные спектры внутренней квантовой эффективности на активную площадь представлены на рис. 2.

По фотоэлектрическим свойствам полученные СЭ не уступают гетероструктурам $\mathrm{GaP} / \mathrm{Si}$, выращенным методом MBE с использованием высокотемпературного отжига (порядка $900^{\circ} \mathrm{C}$ ) на аналогичных подложках $\mathrm{Si}$ [14]. В данной работе температура на всех этапах изготовления СЭ не превышала $650^{\circ} \mathrm{C}$. При этих температурах в процессе эпитаксиального роста, согласно результатам численного расчета, не происходит диффузии фосфора из $\mathrm{GaP}$ в $\mathrm{Si}$ до уровня легирования подложки. Таким образом, для анализа свойств структур применима модель резкого анизотипного гетероперехода $n$ - $\mathrm{GaP} / p$-Si. В этом случае дефекты, образовавшиеся на границе раздела и в приповерхностной области $\mathrm{Si}$, оказывают основное влияние на напряжение холостого хода $V_{o c}[12]$. Использование аргоновой плазмы в процесce PEALD-роста нуклеационного слоя GaP приводит к 


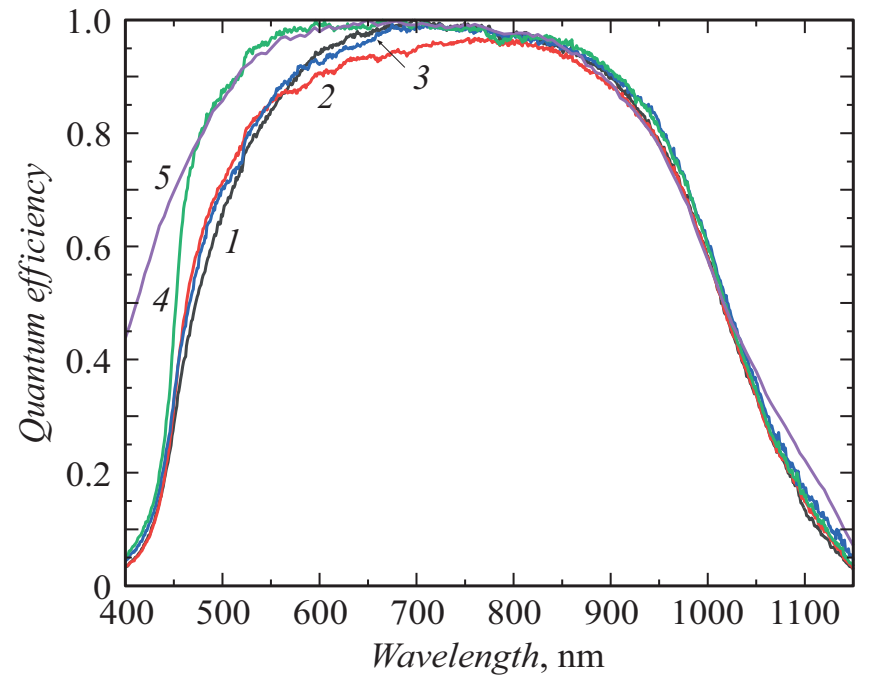

Pис. 2. Спектры внутренней квантовой эффективности на активную площадь для исследуемых структур (1-4) и для исходной структуры, полученной только методом PEALD (5).

снижению значения $V_{o c}$, что, вероятно, связано с формированием радиационных дефектов в приповерхностной области $\mathrm{Si}$, сохраняющихся после газофазной эпитаксии при температуре $600^{\circ} \mathrm{C}$, причем их концентрация не уменьшается и после роста при более высокой температуре $\left(650^{\circ} \mathrm{C}\right)$, на что указывает отсутствие изменения $V_{o c}$. Для структур, полученных без использования плазмы Ar, достигнуты более высокие значения $V_{o c}$, что свидетельствует, как минимум, о меньшей концентрации радиационных дефектов. Повышение температуры MOVPE приводит к росту $V_{o c}$ до значений, предельно достигаемых на данных подложках для структур $\mathrm{GaP} / \mathrm{Si}$, полученных только с помощью PEALD в режиме с минимальной мощностью плазмы, не приводящем к формированию радиационных дефектов [15]. В этом режиме происходит рост аморфных слоев $\mathrm{GaP}$, что, несмотря на лучшие фотоэлектрические свойства исходных структур, не позволяет использовать его для роста нуклеационных слоев.

Длинноволновый край спектров квантовой эффективности, определяемый рекомбинационными потерями в

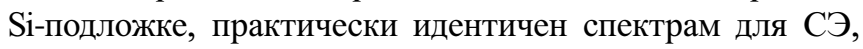
полученных только с помощью PEALD при $390^{\circ} \mathrm{C}$ и имеющих аналогичный тыльный контакт. Данный факт указывает на отсутствие деградации объемного времени жизни неосновных носителей заряда в подложке $p$-Si в процессе газофазной эпитаксии. Коротковолновая область спектров квантовой эффективности определяется поглощением в слое $\mathrm{GaP}$, и наблюдаемое различие связано с разницей в его толщине. Для двухпереходных СЭ спад фоточувствительности в коротковолновой области из-за поглощения в $\mathrm{GaP}$ не имеет значения, так как длинноволновый край фотоактивного материала верхнего перехода должен быть в районе $0.7 \mu \mathrm{m}$.
Таким образом, предлагаемый подход, заключающийся в росте нуклеационного слоя GaP с помощью PEALD без in situ обработки в плазме $\mathrm{Ar}$ и последующем росте с использованием технологии MOVPE, может быть использован для формирования нижнего перехода $n$-GaP/p-Si многопереходных СЭ на основе $\mathrm{A}_{3} \mathrm{~B}_{5} / \mathrm{Si}$ при температурах, не превышающих $650^{\circ} \mathrm{C}$.

\section{Финансирование работы}

Представленные в работе исследования проведены при финансовой поддержке Российского научного фонда (проект № 17-19-01482).

\section{Конфликт интересов}

Авторы заявляют, что у них нет конфликта интересов.

\section{Список литературы}

[1] A. De Vos, J. Phys. D: Appl. Phys., 13, 839 (1980). DOI: $10.1088 / 0022-3727 / 13 / 5 / 018$

[2] J.P. Connolly, D. Mencaraglia, C. Renard, D. Bouchier, Prog. Photovolt.: Res. Appl., 22, 810 (2014). DOI: 10.1002/pip.2463

[3] T. Soga, T. Jimbo, M. Umeno, Appl. Phys. Lett., 63, 2543 (1993). DOI: 10.1063/1.110427

[4] A.S. Gudovskikh, K.S. Zelentsov, A.I. Baranov, D.A. Kudryashov, I.A. Morozov, E.V. Nikitina, J.-P. Kleider, Energy Procedia, 102, 56 (2016).

DOI: $10.1016 /$ j.egypro.2016.11.318

[5] H. Wagner, T. Ohrdes, A. Dastgheib-Shirazi, B. Puthen-Veettil, D. König, P.P. Altermatt, J. Appl. Phys., 115, 044508 (2014). DOI: $10.1063 / 1.4863464$

[6] A.S. Gudovskikh, A.V. Uvarov, I.A. Morozov, A.I. Baranov, D.A. Kudryashov, E.V. Nikitina, A.A. Bukatin, K.S. Zelentsov, I.S. Mukhin, A. Levtchenko, S. Le Gall, J.-P. Kleider, J. Renew. Sustain. Energy, 10, 021001 (2018). DOI: 10.1063/1.5000256

[7] M. Feifel, J. Ohlmann, J. Benick, T. Rachow, S. Janz, M. Hermle, IEEE J. Photovolt., 7, 502 (2017). DOI: $10.1109 /$ jphotov.2016.2642645

[8] C. Zhang, N.N. Faleev, L. Ding, M. Boccard, M.I. Bertoni, Z. Holman, in IEEE 43rd Photovoltaic Specialists Conf. (PVSC) (IEEE, 2016), p. 1950. DOI: $10.1109 /$ pvsc.2016.7749966

[9] H. Döscher, S. Brückner, T. Hannappel, J. Cryst. Growth, 318, 563 (2011). DOI: 10.1016/j.jcrysgro.2010.11.087

[10] R. Varache, M. Darnon, M. Descazeaux, M. Martin, T. Baron, D. Muñoz, Energy Procedia, 77, 493 (2015). DOI: 10.1016/j.egypro.2015.07.070

[11] L. Ding, C. Zhang, T.U. Nærland, N. Faleev, C. Honsberg, M.I. Bertoni, Energy Procedia, 92, 617 (2016). DOI: 10.1016/j.egypro.2016.07.027

[12] A.S. Gudovskikh, A.V. Uvarov, I.A. Morozov, A.S. Bukatin, A.I. Baranov, D.A. Kudryashov, N.A. Kalyuzhnyy, S.A. Mintairov, V.I. Zubkov, G.E. Yakovlev, J.-P. Kleider, Phys. Status Solidi A, 217, 1900532 (2019). DOI: $10.1002 /$ pssa.201900532 
[13] A.V. Uvarov, A.S. Gudovskikh, V.N. Nevedomskiy, A.I. Baranov, D.A. Kudryashov, I.A. Morozov, J.-P. Kleider, J. Phys. D: Appl. Phys., 53, 345105 (2020).

DOI: $10.1088 / 1361-6463 / a b 8 b f d$

[14] М.С. Соболев, А.А. Лазаренко, Е.В. Никитина, Е.В. Пирогов, А.С. Гудовских, А.Ю. Егоров, ФТП, 49 (4), 569 (2015).

[15] A.S. Gudovskikh, A.V. Uvarov, I.A. Morozov, A.I. Baranov, D.A. Kudryashov, K.S. Zelentsov, A. Jaffre, S. Le Gall, A. Darga, A. Brezard-Oudot, J.-P. Kleider, Phys. Status Solidi A, 216, 1800617 (2018). DOI: 10.1002/pssa.201800617 